\title{
COPD: 600 Schritte mehr sollst du tun!
}

\author{
Eine Studie hat die minimale zusätzliche körperliche Aktivität ermittelt, mit der \\ COPD-Patienten eine spürbare und klinisch bedeutsame Verbesserung erreichen.
}

_ Bei chronisch obstruktiver Lungenerkrankung (COPD) ist die körperliche Inaktivität ein entscheidender Prädiktor für eine schlechtere Prognose. In einer retrospektive Auswertung wurden nun die Daten von 74 Patienten mit COPD analysiert. Sie waren $66 \pm 7$ Jahre alt und erreichten $48 \pm 22 \%$ des Sollwerts der Einsekundenkapazität $\left(\mathrm{FEV}_{1}\right)$. Vor und nach einer dreimonatigen ambulanten pneumologischen Rehabilitation trugen sie für jeweils eine Woche Aktivitätsmonitore.

Der primäre Outcome-Parameter, die durchschnittliche Schrittzahl pro Tag, stieg nach der Rehabilitation von 3.839 auf 4.644. Mit vier verschiedenen statistischen Verfahren wurde sodann eine „minimal important difference“ (MID) errechnet - die minimale Veränderung, ab welcher der Patient eine spürbare Verbesserung erreicht. Diese lag im Bereich von 600 bis 1.100 Schritten.

Die klinische Relevanz dieser MID spiegelte sich in der Zeit bis zur ersten Hospitalisation nach der Rehabilitation wider. Patienten, die ihre körperliche Aktivität mindestens um die MID steigerten, erlitten innerhalb der ersten 24 Monate signifikant weniger schwere Exazerbationen mit Hospitalisation.

- Demeyer H, Burtin C, Hornikx M et al. The Minimal Important Difference in Physical Activity in Patients with COPD. PLoS One. 2016 Apr 28;11(4):e0154587

\section{KOMMENTAR}

Dies ist der erste Versuch, eine MID für körperliche Aktivität nach einer pneumologischen Rehabilitation zu definieren. Bislang konnte man Veränderungen der körperlichen Aktivität nur schwer einordnen, da eine aussagekräftige Bezugsgröße fehlte. Für das Design künftiger Studien sind die Ergebnisse also wertvoll. In der Studie wurde die Schrittzahl als Äquivalent für die körperliche Aktivität gewählt, was sowohl Vor-als auch Nachteile hat, die uns auch in der Versorgungspraxis begegnen. Ein Nachteil ist sicherlich, dass die tatsächliche Schrittzahl von einigen Aktivitätsmonitoren erheblich unterschätzt wird - vor allem bei Patienten, die sehr langsam oder mit einem Rollator gehen. Der Vorteil liegt jedoch darin, dass die Schrittzahl ein sehr einfach zu messender Parameter ist. Zudem ist sie im Unterschied zu eher wissenschaftlich validierten Parametern wie dem metabolischen Äquivalent oder dem Physical Activity Level (PAL) gerade für Patienten verständlicher und "greifbarer".

Aus statistischer Sicht lässt sich noch monieren, dass für die Schrittzahl keine ausreichenden Korrelationen zu patientenrelevanten Parametern wie der Sechs-Minuten-Gehteststrecke oder der Lebensqualität vorlagen. Die MID musste deshalb mit rein statistischen Verfahren berechnet werden.

Doch was heißt das nun für die Praxis? Gilt die gleiche "starre" MID von 600 bis 1.100 Schritten für Patienten, die 1.000 Schritte pro Tag oder 10.000 Schritte pro Tag laufen? In diesem Kontext ist wie bei allen MIDs nicht ausgeschlossen, dass der einzelne Patient bei einer
Veränderung seiner Schrittzahl deutlich unter oder über der MID einen positiven Effekt verspürt. MID werden immer für Gruppen berechnet und dürfen streng genommen auch nur für Kohorten angewandt werden. Trotzdem lässt sich der positive Effekt der erhöhten Schrittzahl nicht wegdiskutieren.

In jedem Fall trägt diese erste Studie zur Berechnung einer MID für körperliche Aktivität wesentlich zur COPDForschung bei - und zeigt einen Schwellenwert für ein reduziertes Hospitalistionsrisiko auf.

Dr. phil. R. Glöckl

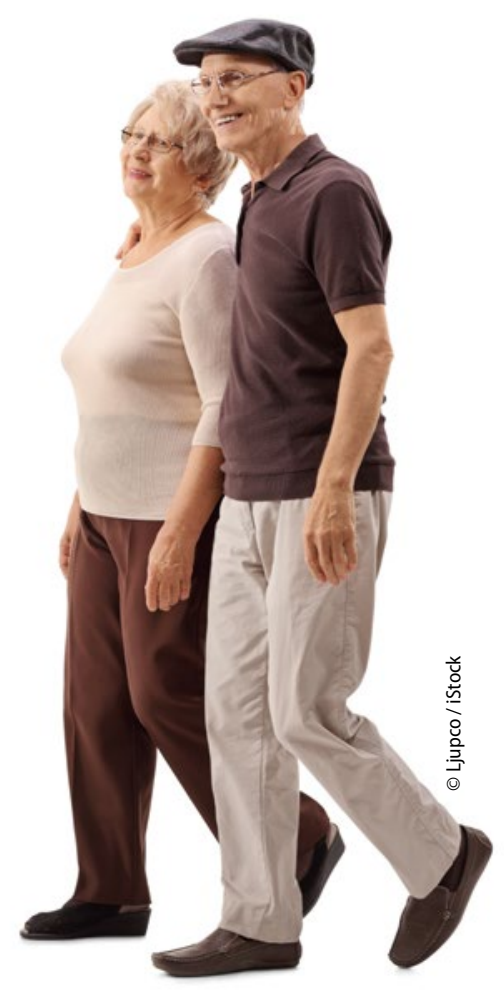

ANZEIGE

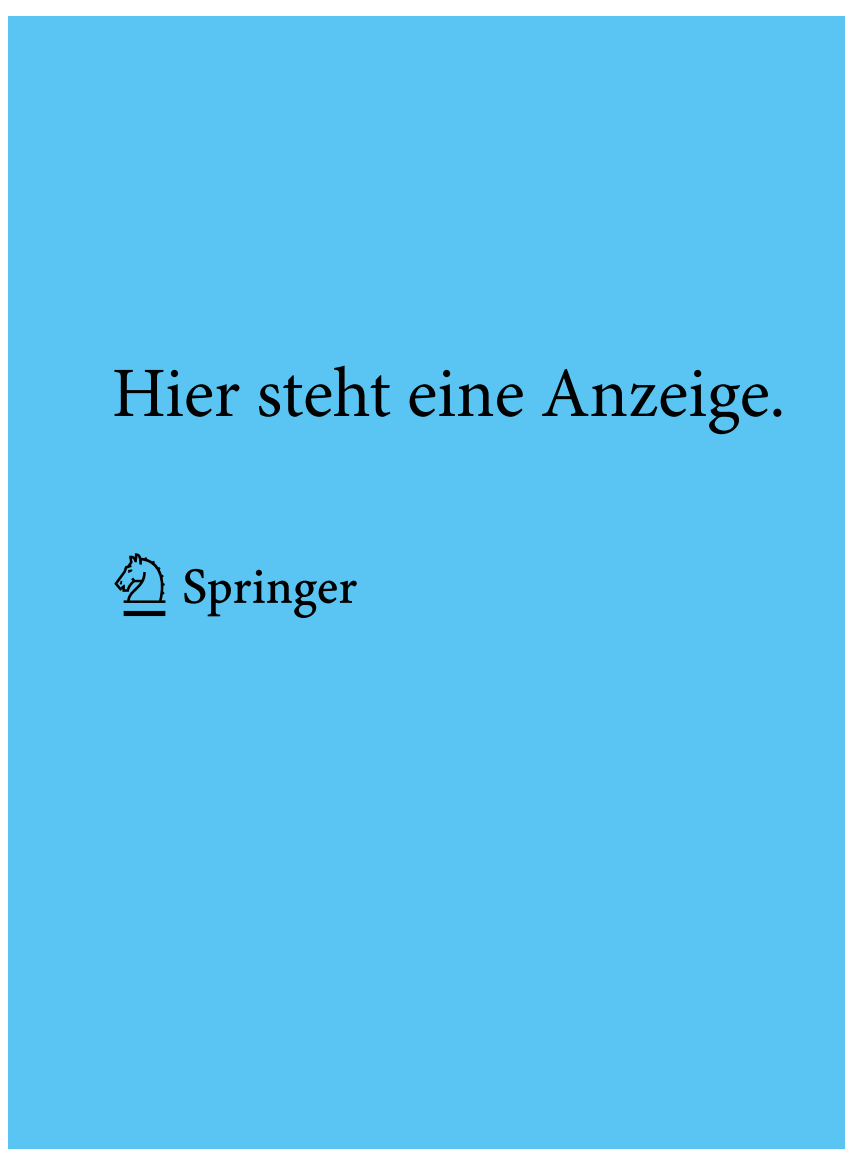

